\title{
SZEGÖ KERNEL, REGULAR QUANTIZATIONS AND SPHERICAL CR-STRUCTURES
}

\author{
CLAUDIO AREZZO, ANDREA LOI, AND FABIO ZUDDAS
}

\begin{abstract}
We compute the Szegö kernel of the unit circle bundle of a negative line bundle dual to a regular quantum line bundle over a compact Kähler manifold. As a corollary we provide an infinite family of smoothly bounded strictly pseudoconvex domains on complex manifolds (disk bundles over homogeneous Hodge manifolds) for which the log-terms in the Fefferman expansion of the Szegö kernel vanish and which are not locally CR-equivalent to the sphere. We also give a proof of the fact that, for homogeneous Hodge manifolds, the existence of a locally spherical CR-structure on the unit circle bundle alone implies that the manifold is biholomorphic to a projective space. Our results generalize those obtained by M. Engliš and G. Zhang in [12 for Hermitian symmetric spaces of compact type.
\end{abstract}

\section{INTRODUCTION}

Let $(L, h)$ be a positive Hermitian line bundle over a compact Kähler manifold $(M, g)$ of complex dimension $n$, such that $\operatorname{Ric}(h)=\omega_{g}$, where $\omega_{g}$ denotes the Kähler form associated to $g$ and $\operatorname{Ric}(h)$ is the two-form on $M$ whose local expression is given by $\operatorname{Ric}(h)=-\frac{i}{2} \partial \bar{\partial} \log h(\sigma(x), \sigma(x))$, for a trivializing holomorphic section $\sigma: U \rightarrow L \backslash\{0\}$. In the quantum mechanics terminology the pair $(L, h)$ is called a geometric quantization of $\left(M, \omega_{g}\right)$ and $L$ the quantum line bundle. Consider the negative Hermitian line bundle $\left(L^{*}, h^{*}\right)$ over $(M, g)$ dual to $(L, h)$ and let $D \subset L^{*}$ be the unit disk bundle over $M$, i.e.

$$
D=\left\{v \in L^{*} \mid \rho(v):=1-h^{*}(v, v)>0\right\}
$$

2000 Mathematics Subject Classification. 53C55; 58C25; 58F06.

Key words and phrases. Szegö kernel, Ramadanov conjecture; regular quantizations; TYZ asymptotic expansion; log-term; CR-structures.

The first author was supported by the M.I.U.R. Project "Geometric Properties of Real and Complex Manifolds" and by ESF within the program "Contact and Symplectic Topology". 
It is not hard to see (and well-known) that the condition $\operatorname{Ric}(h)=\omega_{g}$ implies that $D$ is a strongly pseudoconvex domain in $L^{*}$ with smooth boundary $X=\partial D=\left\{v \in L^{*} \mid \rho(v)=0\right\}$. X will be called the unit circle bundle.

Consider the separable Hilbert space $\mathcal{H}^{2}(X)$ consisting of all holomorphic functions $f: L^{*} \rightarrow \mathbb{C}$ with scalar product given by $\int_{X} f \bar{g} d \mu$ where $d \mu=$ $\alpha \wedge(d \alpha)^{n}$ and

$$
\alpha=-i \partial \rho_{\mid X}=i \bar{\partial} \rho_{\mid X}
$$

is the standard contact form on $X$ associated to the strongly pseudoconvex domain $D$.

Let $\left\{f_{j}\right\}_{j=1, \ldots}$ be an orthonormal basis of $\mathcal{H}^{2}(X)$, i.e.

$$
\int_{X} f_{j} \bar{f}_{k} d \mu=\delta_{j k}
$$

The Szegö kernel of $D$ is defined by:

$$
\mathcal{S}(v)=\sum_{j=1}^{+\infty} f_{j}(v) \overline{f_{j}(v)}, v \in D .
$$

A fundamental result of C. Fefferman 2 [13] asserts that there exist $a, b \in$ $C^{\infty}(\bar{D}), a \neq 0$ on $X=\partial D$ such that:

$$
\mathcal{S}(v)=a(v) \rho(v)^{-n-1}+b(v) \log \rho(v), v \in D
$$

where $\rho(v)=1-h^{*}(v, v)$ is the defining function of $D$.

The function $b(v)$ in (2) is called the logarithmic term (log-term from now on) of the Szegö kernel. One says that the log-term of the Szegö kernel of the disk bundle $D \subset L^{*}$ vanishes if $b=0$.

Example 1. The basic example here is the complex projective space $\mathbb{C P}^{n}$ equipped with the Fubini-Study Kähler form $\omega_{F S}, L=O(1)$ the hyperplane bundle and $L^{*}=O(-1)$ the canonical bundle over $\mathbb{C P}^{n}$. The corresponding unit circle bundle $X \rightarrow \mathbb{C} P^{n}$ is CR-equivalent to $S^{2 n+1}$ via the map $S^{2 n+1} \rightarrow$ $X, z \mapsto(\mathbb{C} z, z)$ and the restriction to $X$ of the projection $\pi: L^{*} \rightarrow \mathbb{C P}^{n}$ is the Hopf fibration. Morever, a direct computation shows that the log-term of the Szegö kernel of the disk bundle vanishes. More generally, for any nonnegative integer $m$, one considers the line bundle $L^{m}=O(m)$ over $\mathbb{C P}^{n}$

\footnotetext{
${ }^{1}$ Notice that the holomorphic functions of $L^{*}$ are automatically $L^{2}$-integrable on $X$ due to the compactness of $M$ and of $X$.

${ }^{2}$ Originally proved for the boundary singularities of the Bergman kernel of a strongly pseduconvex domain in $\mathbb{C}^{n}$.
} 
and its dual $L^{* m}=O(-m)$. In this case the unit circle bundle $X$ is CRequivalent to the lens space $S^{2 n+1} / \mathbb{Z}_{m}$. The diffeomorphism this time is given by the map $z \mapsto\left(\mathbb{C} z, \otimes^{m} z\right)$ from the sphere $S^{2 n+1}$ which induces a diffeomorhism form $S^{2 n+1} / \mathbb{Z}_{m}$ onto $X$ (see e.g. [21, p. 542]). Also in this case the log-term of the Szegö kernel of the disk bundle vanishes (this follows by a direct computation or as a very special case of Theorem 2 below).

Therefore the following questions naturally arise.

Question 1. Let $D$ be as above. Suppose that the log-term of the Szegö kernel of $D$ vanishes. Is it the boundary $X$ of $D$ locally CR-equivalent to the sphere?

Question 2. Assume that $X$ is (locally) CR-equivalent to the sphere. Is it true that $M$ is biholomorphic to $\mathbb{C P}^{n}$ ?

Question 1 is a reasonable question since the singularity of the Szegö kernel is determined locally by the CR-structure of the boundary. This question is analogous to Ramadanov's conjecture asserting that if $\Omega \subset \mathbb{C}^{n}$ is a strongly pseudoconvex domain with smooth boundary such that its Bergman kernel has no logarithmic term then $\Omega$ is biholomorphic to the unit ball in $\mathbb{C}^{n}$ 3. Questions 1 and 2 are inspired by (and implicitly contained in) the paper of Z. Lu and G. Tian 24] (see also the next section) and by the work of M. Engliš and G. Zhang [12 who provides a negative answer to Question 1 and a positive answer to Question 2 when the disk bundle $D \subset L^{*}$ arises from a positive line bundle $L$ over an Hermitian symmetric space of compact type. Indeed, in this case he computes the Szego kernel of $D$, proves that the log-term vanishes and that $X=\partial D$ is locally spherical iff $M=\mathbb{C P}^{n}$. In the same paper he asks (Question 4 in [12]) if there were other cases where the log-term of the Szegö kernel of the disk bundle vanishes.

One of the aim of the paper is to show that there are indeed many other cases where this occurs. This is expressed by the following theorem and its corollary which is the first result of the paper (the reader is referred to the next section for details and references on regular quantizations).

\footnotetext{
${ }^{3}$ Observe that in our situation the disk bundle $D$ is not even homotopically equivalent to a disk in $\mathbb{C}^{n}$ since the the homotopy type of the disk bundle is the same of its base (compact) manifold $M$.
} 
Theorem 1. Let $(L, h)$ be a regular quantization of a compact Kähler manifold $(M, \omega)$ and let $X \rightarrow M$ be the unit circle bundle of $\left(L^{*}, h^{*}\right)$. Then the log-term of the Szegö kernel of D vanishes.

Notice that a compact homogeneous Hodge manifold (i.e. a simplyconnected compact and homogeneous Kähler manifold) admits a regular quantization (see next section). Hence, we get the following corollary of Theorem 1 .

Corollary 2. Let $(M, g)$ be a homogeneous Hodge manifold and let $(L, h)$ be the Hermitian line bundle over $M$ such that $\operatorname{Ric}(h)=\omega_{g}$. Then the log-term of the Szegö kernel of the disk bundle $D \subset L^{*}$ vanishes.

It is worth pointing out that it is still an open problem to understand if there exist non-homogeneous Kähler manifolds which admit a regular quantization. Nevertheless the set of compact homogeneous Hodge manifolds strictly includes the set of Hermitian symmetric spaces of compact type and so our corollary provides new examples, different from the Hermitian symmetric spaces, where the log-term vanishes.

Turning our attention to Question 2, we prove that, among all homogeneous spaces, $\mathbb{C P}^{n}$ is the only one for which $X=\partial D$ is locally CR-equivalent to the sphere $S^{2 n+1}$ at some point. This is expressed by the following theorem which represents our second result and generalizes the above mentioned results of M. Engliš and G. Zhang valid for Hermitian symmetric spaces of compact type.

Theorem 3. If $M$ is a homogeneous Hodge manifold and $X$ has a CRstructure locally equivalent to $S^{2 n+1}$ at same point, then $M$ is biholomorphic to the complex projective space and $L$ is a multiple of the tautological line bundle.

We finally point out that the papers of X. Huang ([15], [16], [17]) dealing with the links of complex analytic spaces with an isolated singularity carrying a spherical CR-structure could be useful to attack Question 2 without the assumption of homogeneity. Indeed, by applying Huang's results to the unit circle bundle of an embedded projective manifold $M$, seen as a link of the affine cone over $M$, one obtains some informations on the CR-structure of the cone and consequently on the manifold $M$. Unfortunately, these informations seem not to be enough to provide a positive answer to Question 2 . 
The paper contains other two sections. In the first one we recall the basic material on Kempf distortion function, TYZ expansion and regular quantizations while the last section is dedicated to the proof of Theorem 1 and Theorem 3 ,

\section{Kempf Distortion function, Szegö KeRnel And REgular QUANTIZATIONS}

Let $(L, h)$ be a positive Hermitian line bundle over a compact Kähler manifold $(M, g)$ of complex dimension $n$, such that $\operatorname{Ric}(h)=\omega_{g}$ as in the introduction. Let $m \geq 1$ be an integer and consider the Kempf distortion function associated to $m g$, i.e.

$$
T_{m g}(x)=\sum_{j=0}^{d_{m}} h_{m}\left(s_{j}(x), s_{j}(x)\right) .
$$

where $h_{m}$ is an hermitian metric on $L^{m}$ such that $\operatorname{Ric}\left(h_{m}\right)=m \omega_{g}$ and $s_{0}, \ldots, s_{d_{m}}, d_{m}+1=\operatorname{dim} H^{0}\left(L^{m}\right)$ is an orthonormal basis of $H^{0}\left(L^{m}\right)$ (the space of holomorphic sections of $L^{m}$ ) with respect to the $L^{2}$-scalar product

$$
\langle s, t\rangle_{m}=\int_{M} h_{m}(s(x), t(x)) \frac{\omega_{g}^{n}(x)}{n !}, s, t \in H^{0}\left(L^{m}\right) .
$$

(In the quantum geometric context $m^{-1}$ plays the role of Planck's constant, see e.g. [2]).

As suggested by the notation this function depends only on the Kähler metric $g$ and on $m$ and not on the orthonormal basis chosen.

The function $T_{m g}$ has appeared in the literature under different names. The earliest one was probably the $\eta$-function of J. Rawnsley [26] (later renamed to $\theta$ function in [10]), defined for arbitrary (not necessarily compact) Kähler manifolds, followed by the distortion function of G. R. Kempf [19] and S. Ji [18, for the special case of Abelian varieties and of S. Zhang [29] for complex projective varieties. The metrics for which $T_{g}$ is constant (i.e. $T_{m g}$ is constant for $m=1$ ) were called critical in [29] and balanced in [11] (see also [4]) where S. Donaldson studies the link between the existence and uniqueness of balanced metrics in a fixed cohomology class and constant scalar curvature metrics.

One can give a quantum-geometric interpretation of $T_{m g}$ as follows. Take $m$ sufficiently large such that for each point $x \in M$ there exists $s \in H^{0}\left(L^{m}\right)$ non-vanishing at $x$ (such an $m$ exists by standard algebraic geometry methods and corresponds to the free-based point condition in Kodaira's theory). 
Consider the so called coherent states map, namely the holomorphic map of $M$ into the complex projective space $\mathbb{C} P^{d_{m}}$ given by:

$$
\varphi_{m}: M \rightarrow \mathbb{C} P^{d_{m}}: x \mapsto\left[s_{0}(x): \cdots: s_{d_{m}}(x)\right] .
$$

One can prove (see, e.g. [3]) that

$$
\varphi_{m}^{*} \omega_{F S}=m \omega_{g}+\frac{i}{2} \partial \bar{\partial} \log T_{m g}
$$

where $\omega_{F S}$ is the Fubini-Study form on $\mathbb{C} P^{d_{m}}$, namely the Kähler form which in homogeneous coordinates $\left[Z_{0}, \ldots, Z_{d_{m}}\right]$ reads as $\omega_{F S}=\frac{i}{2} \partial \bar{\partial} \log \sum_{j=0}^{d_{m}}\left|Z_{j}\right|^{2}$. Since the equation $\partial \bar{\partial} f=0$ implies that $f$ is constant, it follows by (5) that a metric $m g$ is balanced if and only if it is projectively induced (via the coherent states map). Recall that a Kähler metric $g$ on a complex manifold $M$ is projectively induced if there exists a positive integer $N$ and a holomorphic map $\psi: M \rightarrow \mathbb{C} P^{N}$, such that $\psi^{*} g_{F S}=g$ (the author is referred to the seminal paper of E. Calabi [9] for more details on the subject). Not all Kähler metrics are balanced or projectively induced. Nevertheless, G. Tian [28] and W. D. Ruan [27] solved a conjecture posed by Yau by showing that $\frac{\varphi_{m}^{*} g_{F S}}{m} C^{\infty}$-converges to $g$. In other words, any polarized metric on a compact complex manifold is the $C^{\infty}$-limit of (normalized) projectively induced Kähler metrics. S. Zelditch [30] generalized the Tian-Ruan theorem by proving a complete asymptotic expansion (called TYZ expansion) in the $C^{\infty}$ category, namely

$$
T_{m g}(x) \sim \sum_{j=0}^{\infty} a_{j}(x) m^{n-j},
$$

where $a_{j}(x), j=0,1, \ldots$, are smooth coefficients with $a_{0}(x)=1$. More precisely. for any nonnegative integers $r, k$ the following estimate holds:

$$
\left\|T_{m g}(x)-\sum_{j=0}^{k} a_{j}(x) m^{n-j}\right\|_{C^{r}} \leq C_{k, r} m^{n-k-1},
$$

where $C_{k, r}$ are constants depending on $k, r$ and on the Kähler form $\omega_{g}$ and $\|\cdot\|_{C^{r}}$ denotes the $C^{r}$ norm in local coordinates. Later on, Z. Lu [23] (see also [22]), by means of Tian's peak section method, proved that each of the coefficients $a_{j}(x)$ in (6) is a polynomial of the curvature and its covariant derivatives at $x$ of the metric $g$ which can be found by finitely many algebraic operations. Furthermore, he explicitely computes $a_{j}$ with $j \leq 3$.

Notice that prescribing the values of the coefficients of the TYZ expansion gives rise to interesting elliptic PDEs as shown by Z. Lu and G. Tian [24]. 
The main result obtained there is that if the log-term of the Szegö kernel of the unit disk bundle over $M$ defined in the introduction vanishes then $a_{k}=0$, for $k>n$ ( $n$ being the complex dimension of $M$ ). Moreover Z. Lu has conjectured (private communication) that the converse is true, namely if $a_{k}=0$, for $k>n$ then the log-term vanishes.

In this paper we are interested in those Kähler manifolds admitting a regular quantization. A Kähler manifold $(M, g)$ admits a regular quantization if there exists a positive Hermitian line bundle $(L, h)$ as above such that the Kempf distortion $T_{m g}(x)$ is a constant $T_{m g}$ (depending on $m$ ) for all non-negative integer $m \geq 1$. In other words $(M, g)$ admits a regular quantization iff the family of Kähler metrics $m g$ is balanced for all $m$. Regular quantizations play a prominent role in the study of Berezin quantization of Kähler manifolds (see [2] and [4] and reference therein). From our point of view we are interested to the following two facts:

- if a Kähler manifold $(M, g)$ is homogeneous, i.e. the group of holomorphic isometries acts transitively on it, then $(M . g)$ admits a regular quantization (see, e.g. [2, Theorem 5.1] for a proof).

- if a Kähler manifold admits a regular quantization then it follows by the very definition of Kempf distortion function that

$$
T_{m g}(x)=T_{m g}=\frac{h^{0}\left(L^{m}\right)}{V(M)}
$$

and so by Riemann-Roch theorem $T_{m g}$ is a monic polynomial in $m$ of degree $n$.

Thus in the regular case (and hence in the homogeneous case) the TYZ expansion is finite (being a polynomimal), the coefficients $a_{k}$ are constants for all $k$ and $a_{k}=0$ for $k>n$. Hence in the light of Z. Lu conjecture mentioned above one could believe that the Szegö kernel of the disk bundle has vanishing log-term in the regular situation. Our main Theorem 1 shows that this is indeed the case.

\section{The PRoOfs of THE MAIN RESUlts}

Proof of Theorem 11. First notice that the Hardy space $\mathcal{H}^{2}(X)$ defined in the introduction admits the Fourier decomposition into irreducible factors with respect to the natural $S^{1}$-action. More precisely,

$$
\mathcal{H}^{2}(X)=\oplus_{m=0}^{+\infty} \mathcal{H}_{m}^{2}(X)
$$


where $\mathcal{H}_{m}^{2}(X)=\left\{f \in \mathcal{H}^{2}(X) \mid f(\lambda v)=\lambda^{m} f(v), \lambda \in S^{1}\right\}$. S. Zelditch 30] shows that the map $s \in H^{0}\left(L^{m}\right) \mapsto \hat{s} \in \mathcal{H}_{m}^{2}(X)$ given by:

$$
\hat{s}(v)=v^{\otimes m}(s(x)), v \in X, x=\pi(v), \pi: L^{*} \rightarrow M
$$

is an isometry between $H^{0}\left(L^{m}\right)$ and $\mathcal{H}_{m}^{2}(X)$. Moreover, it not hard to see that, for every $v \in X, 4$

$$
\mathcal{S}_{m}(v):=\sum_{j=0}^{d_{m}} \hat{s}_{j}(v) \overline{\hat{s}_{j}(v)}=\sum_{j=0}^{d_{m}} h_{m}\left(s_{j}(x), s_{j}(x)\right)=T_{m g}(x), x=\pi(v) \in M,
$$

where $T_{m g}(x)$ is the Kempf distortion function. Thus

$$
\mathcal{S}(v)=\sum_{m=0}^{+\infty} \mathcal{S}_{m}(v)=\sum_{m=0}^{+\infty}\left(h_{*}(v, v)\right)^{m} T_{m g}(x), v \in D, x=\pi(v) .
$$

Since, by assumption, the quantization $(L, h)$ of $\left(M, \omega_{g}\right)$ is regular one gets by (8) :

$$
\mathcal{S}(v)=\sum_{m=0}^{+\infty} \frac{h^{0}\left(L^{m}\right)}{V(M)}\left(h_{*}(v, v)\right)^{m},
$$

where $V(M)=\int_{M} \frac{\omega_{g}^{n}}{n !}$ denotes the volume of $M$.

As we have already observed in the previous section, by Riemann-Roch $h^{0}\left(L^{m}\right)$ is a monic polynomial in $m$ of degree $n$ so it can be written as linear combination of the binomial coefficients $C_{k}^{m+k}=\frac{(m+k) !}{m ! k !}$, namely

$$
h^{0}\left(L^{m}\right)=\sum_{k=0}^{n} d_{k} C_{k}^{m+k}, d_{n}=n !
$$

Hence

$$
\mathcal{S}(v)=\frac{1}{V(M)} \sum_{k=0}^{n} d_{k} \sum_{m=0}^{\infty} C_{k}^{m+k}\left(h_{*}(v, v)\right)^{m} .
$$

By using the fact that

$$
\sum_{m=0}^{\infty} C_{k}^{m+k} x^{m}=\frac{1}{(1-x)^{k+1}}, 0<x<1
$$

and $\rho(v)=1-h^{*}(v, v)$ one gets:

$$
\mathcal{S}(v)=\frac{1}{V(M)} \sum_{k=0}^{n} d_{k}[\rho(v)]^{-k-1},
$$

\footnotetext{
${ }^{4}$ It is worth pointing out that is exactly formula (9) together with Boutet de MonvelSjöstrand parametrix for the Szegö kernel which allows S. Zelditch [30] to get the TYZ expansion of Kempf's distortion function.
} 
Therefore

$$
\mathcal{S}(v)=a(v) \rho(v)^{-n-1}
$$

where

$$
a(v)=V(M)^{-1} n !+V(M)^{-1} \sum_{k=0}^{n-1} d_{k}[\rho(v)]^{n-k} .
$$

It then follows (cfr. (2) above) that the log-term of the Szegö kernel of the unit disk bundle $D \subset L^{*}$ vanishes and we are done.

In order to prove Theorem 3 we need the following cohomological lemma.

Lemma 4. Let $(M, \omega)$ be a homogeneous Hodge manifold. Assume that the Betti numbers of $M$ satisfy $b_{2 j-2}=b_{2 j}$ for $j=1, \ldots, n$, where we are taking the dimension of the corresponding cohomology groups with real coeffcients. Then $M$ is biholomorphic either to $\mathbb{C} P^{n}$ or to the complex odd quadric

$Q_{n}=\left\{\left[Z_{0}, \ldots, Z_{n+1}\right] \in \mathbb{C} P^{n+1} \mid Z_{0}^{2}+\cdots+Z_{n+1}^{2}=0\right\}, n=2 p-1, n>1$.

Proof. Let us recall that a compact homogeneous Hodge manifold $M$ can be always realized as a flag manifold $G / K$, where $G$ is a semisimple Lie group and $K$ is the stabilizer of an element $x \in \operatorname{Lie}(G)$ for the adjoint action. Such a manifold can be described combinatorially by a painted Dynkin diagram $\Gamma$, that is a Dynkin diagram of a complex semisimple Lie group (see for example [20]) with some nodes painted in black so that by deleting the black nodes from $\Gamma$ one gets the Dynkin diagram of the semisimple part of $K$. One also endows the diagram with an equipment, that is a bjection between the nodes of $\Gamma$ and a basis $\Delta=\left\{\alpha_{1}, \ldots, \alpha_{m}\right\}$ of the root system $R$ of $\operatorname{Lie}(G)$, so that the elements of $\Delta$ associated to the white nodes are a basis of the root system $R_{K}$ of the semisimple part of $\operatorname{Lie}(K)$. In the following, we will call black roots the elements of $R \backslash R_{K}$. By definition of basis, every $\alpha \in R$ can be written as a combination $\alpha=\sum_{i=1}^{m} k_{i} \alpha_{i}$, where the $k_{i}$ 's belong to $\mathbb{Z}$ and are either all non-negative or all non-positive. A non-zero root such that $k_{i} \geq 0$ for every $i=1, \ldots, m$ is called a positive root. The set of positive roots will be denoted $R^{+}$. For every $\alpha=\sum_{i=1}^{m} k_{i} \alpha_{i} \in R^{+}$, the height of $\alpha$ is the integer $h(\alpha)=\sum_{i=1}^{m} k_{i}$. Let $R_{K}^{+}=R_{K} \cap R^{+}$. The following formula for the Poincaré polynomial of $G / K$ can be found in [1]:

$$
P\left(G / K, t^{1 / 2}\right)=\prod_{\alpha \in R^{+} \backslash R_{K}^{+}} \frac{1-t^{h(\alpha)+1}}{1-t^{h(\alpha)}} .
$$


In order to prove the lemma we will use (10) to determine all the painted Dynkin diagrams for which

$$
P\left(G / K, t^{1 / 2}\right)=c+c t+c t^{2}+\cdots+c t^{n}
$$

First, notice that by evaluating both members of (11) at $t=0$ we get $c=1$. We shall now prove by induction on $m$ that, under the assumptions, for every $1 \leq m \leq n$ there exists exactly one root in $R^{+} \backslash R_{K}^{+}$of height $m$. In order to do that, let us denote $f_{\alpha}=\frac{1-t^{h(\alpha)+1}}{1-t^{h(\alpha)}}$ for every $\alpha \in R^{+} \backslash R_{K}^{+}$. If $k=\sharp\left(R^{+} \backslash R_{K}^{+}\right)$, we then have $P(t)=P\left(G / K, t^{1 / 2}\right)=\prod_{i=1}^{k} f_{\alpha_{i}}(t)$.

By a straight calculation, one easily checks that the i-th derivative of $f_{\alpha}$ at $t=0$ vanishes for $0<i<h(\alpha)$ and equals $i$ ! for $i=h(\alpha)$. By (11) we have $P^{\prime}(0)=\sum_{i=1}^{k} f_{\alpha_{i}}^{\prime}(0)=1$, which clearly implies the claim for $m=1$. Now, let us assume that the claim is true for $m=1, \ldots, s-1$. Then, up to reordering the roots, we have

$$
P(t)=\left(1+t+\cdots t^{s-1}\right) \prod_{i=s}^{k} f_{\alpha_{i}}(t)
$$

where $h\left(\alpha_{i}\right) \geq s$ for every $i=s, \ldots, k$. This implies that

$$
P^{(s)}(0)=\left.\frac{d^{s}}{d t^{s}}\right|_{t=0} \prod_{i=s}^{k} f_{\alpha_{i}}(t)=\sum_{i_{s}+\cdots+i_{k}=s} \frac{s !}{i_{s} ! \cdots i_{k} !} f_{\alpha_{s}}^{\left(i_{s}\right)}(0) \cdots f_{\alpha_{k}}^{\left(i_{k}\right)}(0)
$$

and then, by (11),

$$
\sum_{i_{s}+\cdots i_{k}=s} \frac{1}{i_{s} ! \cdots i_{k} !} f_{\alpha_{s}}^{\left(i_{s}\right)}(0) \cdots f_{\alpha_{k}}^{\left(i_{k}\right)}(0)=1
$$

But, since $h\left(\alpha_{j}\right) \geq s$ and $i_{j} \leq s$ for every $j=s, \ldots, k$, we get $\frac{1}{s !} \sum_{j=s}^{k} f_{\alpha_{j}}^{(s)}(0)=$ 1 , which implies the claim for $m=s$ and then concludes the proof by induction.

In particular, for $m=1$, by definition of height it follows that the Dynkin diagram of $G / K$ must have only one black vertex. Thus, we are left with determining the position of this vertex in the diagram. More precisely, one can conclude the proof by showing that, for each of the complex semisimple Lie algebras, the only painted diagrams with only one black vertex and which do not admit two distinct black positive roots having the same height correspond either to $\mathbb{C} P^{n}$ or to the complex odd quadric. This can be obtained by a careful checking of the list of the set of roots $R$ and a basis $\Delta$ 
for each of the complex semisimple Lie algebras (see, for example, Appendix $\mathrm{C}$ of [20]). The details are left to the reader.

Proof of Theorem [3. If $X$ were locally CR-equivalent to $S^{2 n+1}$ at same point, then, by homogeneity, this would be true at every point, i.e. $X$ would be spherical. Since $X$ is compact and homogeneous, Proposition 5.1 in Burns and Shnider [8] would then imply that $X$ is diffeomorphic to a lens space $S^{2 n+1} / \mathbb{Z}_{m}$ for some $m$. Therefore, by a Gysin sequence argument (see e.g. the proof of Corollary 3.7 in [12]) one gets that the Betti numbers of $M$ must satisfy the relations $b_{2 j-2}=b_{2 j}$ for $j=1, \ldots, n$. By Lemma $4 M$ is then biholomorphic either to $\mathbb{C P}^{n}$ or to $Q_{n} \subset \mathbb{C} P^{n+1}, n=2 p-1>1$. The prove will be finished if we show that the case of the odd-dimensional quadric cannot occur when $n>1$. This follows by the fact that the unit circle bundle over $Q_{n}$ is the Stiefel manifold $V_{2}\left(\mathbb{R}^{n+2}\right)$ (see [7, p. 1581]) which is not diffeomorphic to $S^{2 n+1} / \mathbb{Z}_{m}$ for $n>2$ (for example $H^{2}\left(S^{2 n+1} / \mathbb{Z}_{m}, \mathbb{Z}\right)=\mathbb{Z}_{m}$ while $H^{2}\left(V_{2}\left(\mathbb{R}^{n+2}\right), \mathbb{Z}\right)=0$, for $n>2$, see [14] and [6], respectively, for the computation of the $\mathbb{Z}$-cohomolgy ring of the lens spaces and of the Stiefel manifolds). This ends the proof of the theorem.

Remark 5. In the proof of Theorem 3 the relation between the Betti numbers of $M$ (and the homogeneity) allows to deduce that our manifold $M$ is biholomorphic to a complex projective space. We want to point out that there exist complex algebraic surfaces with the same Betti numbers of a complex projective but not biholomorphic to it. These are the celebrated fake projective spaces (see, e.g. [25], for details). Such a Kähler surface $\left(F, g_{F}\right)$ is obtained by taking the quotient of the unit ball $B^{2} \subset \mathbb{C}^{2}$ by a subgroup of biholomorphisms $\Gamma$ of $B^{2}$, i.e. $F=B^{2} / \Gamma$ and the metric $g_{F}$ is the unique (up to homotheties) Kähler-Einstein metric on $F$ with negative scalar curvature whose pull-back to $B^{2}$ is given by the hyperbolic metric $g_{\text {hyp }}$.

Now, consider a geometric quantization $(L, h)$ for $\left(F, g_{F}\right)$ and the corresponding Kempf distortion function $T_{m g_{F}}$. It is not hard to see that the coefficients in the TYZ expansion of $T_{m g_{F}}$ are constants and, moreover, $a_{k}=0$, for $k>2$. Indeed this follows by the fact that, by Lu's theorem the coefficients depend on the curvature and are the same as those of $\left(B^{2}, g_{\text {hyp }}\right)$ for which the computation is well-known. Hence, if one believes the validity of Lu's conjecture mentioned above (see the end of Section 22), $\left(F, g_{F}\right)$ would be an example of projective algebraic surface with the same Betti numbers of the projective space, with vanishing log-term not biholomorphic to $\mathbb{C P}^{2}$. 
Observe that in this case even if all coefficients of TYZ expansion are constants the quantization is not regular and so the vanishing of the log-term cannot be deduced by Theorem 1. Indeed if the quantization were regular then $g_{F}$ would be projectively induced and the same would be true for the metric $g_{h y p}$ on $B^{2}$, in contrast with a well-known result of Calabi [9].

\section{REFERENCES}

[1] E. Akyildiz, On the Factorization of the Poincare polynomial: A Survey, Serdica Math. J. 30 (2004), 1001-1018.

[2] C. Arezzo, A. Loi, Quantization of Kähler manifolds and the asymptotic expansion of Tian-Yau-Zelditch, J. Geom. Phys. 47 (2003), 87-99.

[3] C. Arezzo and A. Loi, Moment maps, scalar curvature and quantization of Kähler manifolds, Comm. Math. Phys. 246 (2004), 543-549.

[4] C. Arezzo, A. Loi, F. Zuddas, On homothetic balanced metrics, Ann. Global Anal. Geom. 41, n. 4 (2012), 473-491.

[5] F. A. Berezin, Quantization, Izv. Akad. Nauk SSSR Ser. Mat. 38 (1974), 1116-1175 (Russian)

[6] A. Borel, Sur la cohomologie des espaces fibres principaux et des espaces homogenes de groupes de Lie compacts, Ann. of Math. 57 (1953), 115-207.

[7] C. P. Boyer, K. Galicki, M. Nakamaye, Einstein metrics on rational homology 7 spheres, Ann. Inst. Fourier, Grenoble (2002), 1569-1584.

[8] D. Burns, S. Shnider, Spherical hypersurfaces in complex manifolds, Invent. Math. 33 (1976), 223-246.

[9] E. Calabi, Isometric Imbeddings of Complex Manifolds, Ann. of Math. 58 (1953), $1-23$.

[10] M. Cahen, S. Gutt, J. H. Rawnsley, Quantization of Kähler manifolds I: Geometric interpretation of Berezin's quantization, JGP. 7 (1990), 45-62.

[11] S. Donaldson, Scalar Curvature and Projective Embeddings, I, J. Diff. Geometry 59 (2001), 479-522.

[12] M. Engliš, G. Zhang, Ramadanov conjecture and line bundles over compact Hermitian symmetric spaces. Math. Z. 264 (2010), no. 4, 901-912.

[13] C. Fefferman, The Bergman kernel and biholomorphic mappings of pseudoconvex domains, Invent. Math. 26 (1974), 1-65.

[14] A. Hatcher, Algebraic Topology, Cambridge University Press, Cambridge 2002.

[15] X. Huang, Isolated complex singularities and their CR links, Sci. China Ser. A 49 (2006), no. 11, 1441 - 1450.

[16] X. Huang, On the mapping problem for algebraic real hypersurfaces in complex spaces of different dimensions, Annales de l'Institut Fourier 44 (1994), 433-463.

[17] X. Huang and S. Ji, Global holomorphic extension of a local map and a Riemann mapping theorem for algebraic domains, Math. Res. Lett 5, 247-260 (1998).

[18] S. Ji, Inequality for distortion function of invertible sheaves on Abelian varieties, Duke Math. J. 58 (1989), 657-667. 
[19] G. R. Kempf, Metrics on invertible sheaves on abelian varieties, Topics in algebraic geometry (Guanajuato) (1989).

[20] A. W. Knapp, Lie groups beyond an introduction, Progress in Mathematics, 140, Birkhaeuser 1996.

[21] M. Komuro, On Atiyah-Patodi-Singer $\eta$-invariant for $S^{1}$ bundles over Riemann surfaces, J. Fac. Sci. Univ. Tokyo Sect. LA Math. 30 (1984) 525-548.

[22] C-J. Liu, Z. Lu, Generalized asymptotic expansions of Tian-Yau-Zelditch, arXiv:0909.4591.

[23] Z. Lu, On the lower order terms of the asymptotic expansion of Tian-Yau-Zelditch, Amer. J. Math. 122 (2000), no. 2, 235-273.

[24] Z. Lu and G. Tian, The log term of Szegö Kernel, Duke Math. J. 125 (2004), 351-387.

[25] G. Prasad and S. Yeung, Arithmetic fake projective spaces and arithmetic fake Grassmannians, Amer. J. Math. 131 (2009), no. 2, 379-407.

[26] J. H. Rawnsley, Coherent states and Kähler manifolds, The Quarterly Journal of Mathematics (1977), 403-415.

[27] W. D. Ruan, Canonical coordinates and Bergmann metrics, Comm. in Anal. and Geom. (1998), 589-631.

[28] G. Tian, On a set of polarized Kähler metrics on algebraic manifolds, J. Diff. Geometry 32 (1990), 99-130.

[29] S. Zhang, Heights and reductions of semi-stable varieties, Comp. Math. 104 (1996), 77-105.

[30] S. Zelditch, Szegö Kernels and a Theorem of Tian, Internat. Math. Res. Notices 6 (1998), 317-331.

Abdus Salam International Center for Theoretical Physics, Strada Costiera 11, Trieste (Italy) and Dipartimento di Matematica, Università di Parma, Parco Area delle Scienze 53/A, Parma (Italy)

E-mail address: arezzo@ictp.it

Dipartimento di Matematica, Università di Cagliari

E-mail address: loi@unica.it

Dipartimento di Matematica e Informatica, Via delle Scienze 206, Udine (ITALY)

E-mail address: fabio.zuddas@uniud.it 